\title{
Factores que favorecen la realización de proyectos investigativos en estudiantes universitarios
}

\author{
Lucia Palacios-Moya ${ }^{1}$, Luis F. Garcés-Giraldo ${ }^{2}$, Alejandro Valencia-Arias ${ }^{3,4^{\star}}$ y Martha Benjumea-Arias ${ }^{5}$ \\ (1) Centro de Investigaciones de Escolme (CIES). Institución Universitaria Escolme. Cl. 50 \#\#40-39 Medellín - Colombia \\ (correo-e: cies@escolme.edu.co) \\ (2) Vicerrectoría de Investigaciones. Corporación Universitaria Americana. Carrera 43 \# 49-57 Medellín - Colombia \\ (correo-e: Igarces@americana.edu.co) \\ (3) Facultad de Ingeniería. Corporación Universitaria Americana. Carrera 43 \# 49-57 Medellín - Colombia \\ (correo-e: javalenciar@gmail.com) \\ (4) Instituto de Investigación, Universidad Católica Los Ángeles de Chimbote, Jirón Tumbes 247, Chimbote 02804, Perú. \\ (5) Facultad de Ciencias Económicas y Administrativas, Departamento de Finanzas, Instituto Tecnológico Metropolitano \\ - ITM, Calle 54 A \#30-01, Medellín - Colombia (correo-e: marthabenjumea@itm.edu.co).
}

* Autor a quien debe ser dirigida la correspondencia.

Recibido Ene. 27, 2021; Aceptado Mar. 23, 2021; Versión final Abr. 18, 2021, Publicado Ago. 2021

\begin{abstract}
Resumen
El propósito de este artículo es analizar los factores que favorecen la intención de llevar a cabo proyectos de investigación por parte de estudiantes universitarios. Se desarrolló una investigación cuantitativa tipo exploratoria-descriptiva y se recolectó la información mediante una encuesta a 141 estudiantes. A partir de las variables del instrumento, se agruparon unos componentes (factores) para aplicar el análisis exploratorio y confirmatorio y así identificar respectivas asociaciones. Se encontró que los aprendizajes que permanecerán a lo largo de la vida del estudiante tienen una influencia positiva frente a la utilidad que este le asigna al trabajo de grado como elemento complementario y necesario en su formación académica. Se concluye que el docente deberá ejercer un papel dinámico y fuertemente activo a la hora de dirigir las sesiones de asesoría de trabajo de grado con el estudiante, ya que, según su experiencia en dichos espacios, será su percepción de utilidad del trabajo de investigación.
\end{abstract}

Palabras clave: proyecto; investigación; educación superior; plan; estudios; trabajo; estudiante universitario

\section{Factors that encourage university students to conduct research projects}

\begin{abstract}
The purpose of this study was to assess the factors that favor the intent of university students to conduct research projects. A quantitative exploratory-descriptive research was developed and 141 students were surveyed. Based on the variables, some components (factors) were grouped to identify links by applying exploratory and confirmatory analyses. The results showed that what students learn while conducting research projects will have a positive influence on their lives. This was dependent on the amount of work students assign to their projects as a complementary and necessary element to reinforce their academic education. It is concluded that professors must exercise a dynamic and strong active role in advisory committees since students' perceptions about their research projects depend on what students experience in advisory committee meetings.
\end{abstract}

Keywords: research project; higher education; curriculum; study; work; university student 


\section{INTRODUCCIÓN}

Los procesos científicos actuales se caracterizan por el marcado vínculo existente entre la ciencia y la tecnología, donde se busca alcanzar desarrollos en dimensiones sociales, políticas y económicas, por lo que estos precisan soportarse en el nuevo conocimiento generado, el cual cobra mayor relevancia cuando su sentido práctico permite su aplicabilidad en la resolución de problemas reales (Vázquez y Manassero, 2017). Es por esto que, durante la última década del siglo XX, los países latinoamericanos han enfocado sus esfuerzos en fortalecer ecosistemas de ciencia y tecnología, a través de los cuales se fomenten dinámicas de innovación, reconociendo en éstas un factor clave para mejorar la competitividad económica de los Estados, posibilitando así su congruencia con las nuevas configuraciones globales que se han dado en materia de desarrollo (Núñez y Montalvo, 2015).

De igual forma, esta situación ha cobrado mayor ahínco en el marco de la que ha sido denominada como "sociedad del conocimiento", sumamente competitiva, en la cual cada vez más el uso de la información, fomento de la innovación e incorporación de las Tecnologías de la Información y la Comunicación -TIC- se han consolidado como elementos cruciales (García, et al., 2020); lo que ha llevado a que se presenten cambios en aspectos laborales y de formación universitaria de los profesionales que harán parte de un mercado global e interconectado, con lo que las Instituciones de Educación Superior -IES- han asumido nuevos retos frente a las formas en que presenta la oferta educativa (Basantes et al., 2017). En este sentido, los procesos de alfabetización científica han cobrado cada día mayor interés, cuyo propósito es propiciar la educación para la ciencia, llevando a la comprensión sobre lo que ésta significa -es decir, desde un enfoque conceptual- y sus implicaciones prácticas -en la forma como opera en la actualidad-; lo cual es aplicado a diversos campos del conocimiento, llevando a su inclusión en los currículos de los diferentes niveles educativos (Vázquez y Manassero, 2017).

Estos procesos cumplen un papel fundamental al incentivar en los individuos un pensamiento crítico, que les permita hacer frente a las problemáticas con las que se enfrentan en la cotidianidad (Soria-Barreto y Cleveland-Slimming, 2020), mejorar la preparación de los estudiantes para seguir carreras en ciencias (Krim et al., 2019) e incluso favorecer el perfil profesional de la comunidad estudiantil (Valencia et al., 2015). Por tanto, habilidades que usualmente han sido consideradas como competencias básicas en los procesos de formación, como la escritura y la lectura, han pasado a convertirse en un compromiso ineludible para que los ciudadanos puedan interactuar de forma crítica con su contexto social, político y ético; permitiéndoles además interpretar las realidades en que se inscriben sus vidas. No en vano, alcanzar una sinergia entre la ciencia, tecnología y la sociedad, fomenta la educación científica de las personas, para construir nuevo conocimiento que a su vez promueva la generación de soluciones a los problemas cada vez más complejos que experimenta el mundo actual (García y Martínez, 2015).

Con este panorama, los diferentes agentes que confluyen al interior de los sistemas educativos cada vez se vuelven más relevantes para el fomento de la ciencia, como es el caso de las universidades, las cuales han adquirido un importante papel en la generación de nuevo conocimiento, así como en su apropiación al contexto en el cual operan; por esto el desarrollo de competencias investigativas requiere de más fuerza en el proceso de enseñanza - aprendizaje, requeridas tanto por docentes e investigadores, como por los estudiantes. De hecho, desde el comienzo del milenio el foco de la educación ha estado dirigido en formar estudiantes con conocimientos científicos (Moeed, 2015).

Al respecto, diferentes estudios se han aproximado a esta temática con diversos agentes de los sistemas educativos, como es el caso de Alarco et al. (2017), quienes implementaron un estudio en Perú para determinar el interés de estudiantes de pregrado por la realización de investigación científica en el campo de medicina, identificando así una intencionalidad superior al $80 \%$, aunque, al explorar los intereses por hacer una carrera en este ámbito se encontró que sólo el 53,9\% pensarían en ello. Además, para el caso de los autores, la diferenciación de intereses por género si fue un asunto relevante, al encontrar mayor intención por aspectos como la escritura de artículos científicos en las mujeres. Ahora bien, desde enfoques cualitativos se identifican otros aportes, como el realizado por Aiquipa et al. (2018), quienes exploraron a través de técnicas de la Teoría Fundamentada la intención de estudiantes de psicología por llevar a cabo un proyecto de tesis, identificando así la confluencia de factores psicológicos, familiares, sociales, culturales y propios de las universidades que inciden en dicha intención. Así, entre otros factores, los autores identifican: el tiempo que demandan las actividades científicas para ser desarrolladas, los gastos financieros, el apoyo provisto por las universidades, los patrones de crianza y sistemas de creencias como factores clave para entender la intención o no de llevar a cabo este tipo de proyectos.

Sin embargo, pese a que ha sido una temática explorada en la región latinoamericana, y que existe una importante disponibilidad de literatura centrada en la experiencia y habilidades de la investigación de los estudiantes de estudiante de STEM (Science, Technology, Engineering and Mathematics), y que está siendo un tema relevante en las carreras relacionadas con las ciencias administrativas (Kirillova y Au, 2020), no se 
identifican estudios en profundidad desde el enfoque cuantitativo en el contexto colombiano, por lo que la propuesta metodológica del estudio aquí expuesto basada en el modelo D de Somers, se convierte en una ventana de oportunidad para fortalecer los conocimientos generados sobre esta temática. Por tanto, se llevó a cabo un estudio cuyo propósito consistió en analizar los factores que favorecen la intención de llevar a cabo proyectos de investigación por parte de estudiantes universitarios en la ciudad de Medellín (Colombia), para el año 2019.

\section{MÉTODO}

Se diseñó una investigación con enfoque cuantitativo bajo un diseño no experimental y de alcance exploratorio-descriptivo, para poder analizar los factores que favorecen la intención de llevar a cabo proyectos de investigación en la población de estudiantes universitarios en la ciudad de Medellín (Colombia) que cumplieran con los siguientes criterios de inclusión: haber participado en semilleros de investigación, haber desarrollado proyectos de corte investigativa bien sea en calidad de auxiliar o como requisito para optar su título tecnológico o profesional, haber participado la construcción del proyecto mínimamente por una duración de 6 meses. De igual forma se aplicó como criterio exclusión cursar un estudio universitario de nivel posgrado (especialidad, maestría, doctorado), tener un estudio de posgrado y que estuviese participando en la fase inicial de la construcción de un proyecto investigativo.

Para seleccionar a la población de estudio se aplicó una muestra no probabilística a conveniencia, obteniendo al final la participación de 141 estudiantes de programas de nivel tecnológico (gestión en finanzas, redes y seguridad informática, mercadotecnia, gestión de empresas de salud, comercio exterior) y profesional (administración de empresas, administración financiera, contaduría pública, negocios internacionales, mercadeo), de 5 y 6 semestre (tecnologías) y de 9 y 10 semestre (profesional).

Como instrumento de recolección de información se diseñó una encuesta, que se formuló apoyándose de una revisión de literatura en bases de datos internacionales con el fin de explorar los hallazgos de otras investigaciones frente a los motivantes de los estudiantes universitarios para formular y participar en proyectos de investigación y así definir las variables que permitieran agrupar, analizar y establecer asociaciones de los factores motivantes. Dicho instrumento estuvo conformado por 36 preguntas dicotómicas, politómicas y de escala Likert bajo la siguiente clasificación 5: Totalmente de acuerdo; 4: de acuerdo; 3: ni de acuerdo, ni en desacuerdo; 2: en desacuerdo y 1: totalmente en desacuerdo; las cuales abordaban variables sociales, demográficas, familiares, académicas, laborales, curriculares, entre otras.

Se realizó un análisis factorial exploratorio el cual es el nombre genérico con que se designa un conjunto de métodos estadísticos multivariados de interdependencia, cuyo propósito principal es identificar una estructura de factores subyacentes a un conjunto amplio de datos con el propósito de obtener una estructura base del modelo teórico a presentar y posteriormente validarlo mediante un análisis factorial confirmatorio. El procedimiento supuso analizar cada conjunto de variables de acuerdo con los siguientes aspectos: en primer lugar, se aplicó el análisis exploratorio a todo el grupo de variables utilizando Máxima Verosimilitud (ML) como procedimiento de extracción de los factores y eligiendo VARIMAX como procedimiento de rotación, para ello se utilizó el software estadístico SPSS. En segundo lugar, se plantea un modelo en el que se busca validar ocho hipótesis, de acuerdo con los factores obtenidos después de efectuar el análisis exploratorio de los datos, finalmente se espera corroborar la estructura factorial de cargas estandarizadas obtenidas para cada constructo a través de la aplicación de análisis factorial confirmatorio. De este modo, se obtuvo que las veintidós variables analizadas se agrupan en seis componentes o factores, tal y como se señalan en la tabla 1.

Tabla 1: Componentes con relación a variables

\begin{tabular}{|c|l|}
\hline Componente & \multicolumn{1}{c|}{ Descripción } \\
\hline Componente 1 (UP) & $\begin{array}{l}\text { Este componente está asociado a las variables relacionadas con el aporte intelectual y } \\
\text { personal que los estudiantes perciben luego de la realización del trabajo de grado, también } \\
\text { está directamente relacionado a las ventajas que les genera la realización de un trabajo de } \\
\text { investigación en su campo laboral, viéndose reflejados estos beneficios en su desempeño en } \\
\text { el contexto empresarial, y entendiendo que, luego de realizado este esfuerzo académico, los } \\
\text { estudiantes se sienten empoderados con capacidad de formular y ejecutar proyectos en el } \\
\text { ámbito científico. }\end{array}$ \\
\hline Componente 2 (RD) & $\begin{array}{l}\text { Las variables agrupadas a este factor corresponden a aquellas que evalúan el rol del docente } \\
\text { en la consecución del trabajo de grado. Cada ítem de este componente mide la percepción } \\
\text { de los estudiantes frente a la atención prestada por el personal docente en su proceso de } \\
\text { construcción del trabajo de grado, así como también se evalúan aspectos relacionados con la } \\
\text { buena empatía y relación con el docente como ruta para conseguir un desarrollo óptimo del } \\
\text { trabajo de investigación. }\end{array}$ \\
\hline
\end{tabular}


Tabla 1: continuación

\begin{tabular}{|l|l|}
\hline Componente & Descripción \\
\hline Componente 3 (FP) & $\begin{array}{l}\text { Las variables contenidas en este componente hacen referencia a la facilidad percibida por el } \\
\text { estudiante de acuerdo con su grado de compatibilidad con el tema de investigación y sus } \\
\text { habilidades. Con el fin de identificar el nivel de satisfacción que los estudiantes experimentan } \\
\text { con su tema de investigación y la facilidad de elección a la hora de determinar la temática } \\
\text { sobre a cuál trabajarán, se realizan preguntas sobre grado de consciencia que se desarrolla } \\
\text { en el estudiante a partir de los cursos universitarios y se indaga sobre la situación familiar y } \\
\text { económica del estudiante para así entender la eficiencia en el desarrollo del proyecto de } \\
\text { grado. }\end{array}$ \\
\hline Componente 5 (HI) & $\begin{array}{l}\text { Asociado a las variables relacionadas con las habilidades investigativas del estudiante, } \\
\text { específicamente en materia de disposición de herramientas para la construcción del proyecto } \\
\text { de grado y la motivación por continuar con estudios de posgrado. }\end{array}$ \\
\hline Componente 6 (AV) & $\begin{array}{l}\text { Este componente está asociado a las variables relacionadas con los aprendizajes para la vida } \\
\text { luego de la realización de un trabajo de grado. }\end{array}$ \\
\hline
\end{tabular}

Una vez abordado este proceso, se llevó a cabo la recolección de información aplicando las encuestas directamente a los estudiantes que estaban desarrollando proyectos de corte investigativo, para ello se solicitó apoyo de los docentes asesores para concretar encuentros en el aula. Al terminar este proceso, las encuestas fueron tabuladas y se creó una base de datos en el programa Microsoft Excel, la cual sirvió como archivo principal para hacer el análisis descriptivo de los datos y el posterior análisis factorial exploratorio y confirmatorio mediante el programa SPSS versión 22.

\section{RESULTADOS}

Durante la primera fase del análisis, se logró constatar que los datos recolectados en la investigación no parecen mostrar información redundante, ya que, las cargas factoriales de las variables observables en su mayoría fueron superiores a 0,6, logrando que el promedio estas para cada constructo obtuviera un valor superior a 0,7 para lograr la convergencia del modelo. Posterior a este análisis, se presenta el modelo de hipótesis a validar. Una vez efectuado el análisis factorial exploratorio se realizó el análisis factorial confirmatorio, para demostrar la validez de la estructura factorial previamente obtenida con el análisis exploratorio y, por consiguiente, la validez de las deducciones teóricas inferidas del mismo. De acuerdo con lo anterior, el procedimiento que se llevó a cabo consistió en la validación de las escalas en las que se midió la información y la fiabilidad del instrumento de medida utilizado.

\section{Validez Convergente}

La validez de las escalas de medida utilizadas junto con cada uno de los constructos y el instrumento en general se comprobó a través del método estadístico análisis factorial confirmatorio, que agrupa procedimientos técnicos y variables para evaluar la multidimensionalidad de un constructo ya que, permite una exploración empírica considerando que el objetivo es seleccionar aquellos ítems que correlacionan mayor con el conjunto de ítems que están midiendo el constructo. Para ello, se debe tener en cuenta que, la fiabilidad del modelo se valora en dos niveles, por un lado, la fiabilidad de los ítems observables; y por otro lado se valora la fiabilidad de los constructos (Calvo-Porral et al. 2013). Ahora bien, para determinar su impacto sobre el modelo, algunos autores han establecido que, una fiabilidad superior a 0.6 es considerada como evidencia de que el modelo es fiable (Bagozzi y Yi, 1988), así mismo, la fiabilidad de los constructos se refiere al grado con que una variable observable refleja un factor, considerándose aceptable un valor superior a 0.7 . Se tiene entonces que, la validez convergente evalúa el grado en que la medida de los ítems que recogen un mismo concepto están correlacionados (Calvo-Porral et al. 2013). Además, en el presente estudio no fue necesario eliminar ningún indicador, dado que su carga factorial estandarizada cumple con los criterios de evaluación establecidos por los autores citados previamente, tal y como se muestra en la tabla 2.

En lo que respecta a los estadísticos correspondientes al estudio de la adecuación de la muestra al modelo son la prueba de esfericidad de Bartlett y la medida de adecuación de la muestra Kaiser-Meyer-Olkin (KMO). La primera, se emplea para contrastar la hipótesis de que la matriz de correlaciones obtenida no es una matriz de identidad (de la Fuente Arias y Justicia, 2003) pues su p debe ser inferior a los niveles críticos 0.05 o 0.01. El modelo planteado presenta valores de Bartlett iguales a cero, por ende, se puede afirmar que existen correlaciones significativas entre las variables. Por otro lado, la segunda, se define como un índice que compara las magnitudes de los coeficientes de correlación observados con las magnitudes de los coeficientes de correlación parciales, y su valor está comprendido entre 0 y 1 , utilizada como medida de adecuación de la muestra, sabiendo que los valores bajos en dicho índice descartan la aplicación de este análisis (de la Fuente 
Arias y Justicia, 2003). Se caracterizan estos valores en una escala que considera a las medidas de KMO próximas a 0.90 como excelentes, a 0.80 como meritorias, a 0.70 como medianas, a 0.60 como mediocres y por debajo a 0.50 como inaceptables (Gómez-Molina et al. 2006). En este sentido, los coeficientes arrojados por el software SPSS para cada uno de los factores, cumple con los criterios mencionados, lo cual indica que es factible realizar la técnica de reducción de datos.

Tabla 2: Cargas factoriales estandarizadas del modelo propuesto.

\begin{tabular}{|c|c|c|c|}
\hline Factor & Ítem & $\begin{array}{l}\text { Cargas } \\
\text { factoriales } \\
\text { estandarizadas }\end{array}$ & $\begin{array}{l}\text { Promedio de } \\
\text { cargas factoriales } \\
\text { estandarizadas }\end{array}$ \\
\hline \multirow{2}{*}{$\begin{array}{l}\text { Aprendizajes para la vida luego de la } \\
\text { realización de un trabajo de grado } \\
\text { (AV) }\end{array}$} & $\mathrm{AV} 1$ & 0.828 & \multirow{2}{*}{0.828} \\
\hline & AV2 & 0.828 & \\
\hline \multirow{3}{*}{$\begin{array}{l}\text { Barreras percibidas por el estudiante } \\
\text { para la culminación del trabajo de } \\
\text { grado (BP) }\end{array}$} & BP1 & 0.702 & \multirow{3}{*}{0.737} \\
\hline & BP2 & 0.727 & \\
\hline & BP3 & 0.781 & \\
\hline \multirow{4}{*}{$\begin{array}{l}\text { Facilidad percibida por el estudiante } \\
\text { de acuerdo con su grado de } \\
\text { compatibilidad con tema de } \\
\text { investigación y sus habilidades (FP) }\end{array}$} & FP1 & 0.747 & \multirow{4}{*}{0.656} \\
\hline & FP2 & 0.545 & \\
\hline & FP3 & 0.647 & \\
\hline & FP4 & 0.685 & \\
\hline \multirow{3}{*}{$\begin{array}{l}\text { Habilidades investigativas del } \\
\text { estudiante }(\mathrm{HI})\end{array}$} & $\mathrm{HI} 1$ & 0.691 & \multirow{3}{*}{0.748} \\
\hline & $\mathrm{HI} 2$ & 0.827 & \\
\hline & $\mathrm{HI} 3$ & 0.726 & \\
\hline \multirow{4}{*}{$\begin{array}{l}\text { Rol del docente en la consecución del } \\
\text { trabajo de grado (RD) }\end{array}$} & RD1 & 0.594 & \multirow{4}{*}{0.763} \\
\hline & RD2 & 0.854 & \\
\hline & RD3 & 0.796 & \\
\hline & RD4 & 0.809 & \\
\hline \multirow{6}{*}{$\begin{array}{l}\text { Utilidad percibida del trabajo de grado } \\
\text { (UP) }\end{array}$} & UP1 & 0.803 & \multirow{6}{*}{0.727} \\
\hline & UP2 & 0.686 & \\
\hline & UP3 & 0.677 & \\
\hline & UP4 & 0.695 & \\
\hline & UP5 & 0.785 & \\
\hline & UP6 & 0.719 & \\
\hline
\end{tabular}

\section{Validez Discriminante}

La validez discriminante se comprobó mediante la corroboración de que el intervalo de confianza en la estimación de la correlación entre cada par de factores no contuviera el valor 1. Como se muestra en la tabla 3 , todos los constructos del modelo propuesto cumplen con dicho criterio y de esta forma se constata que, la validez del instrumento utilizado mide en alto grado aquello que pretendía medir.

Tabla 3: Coeficiente de correlación de Spearman

\begin{tabular}{|c|c|c|c|c|c|c|}
\hline & AV & BP & FP & HI & RD & UP \\
\hline AV & $\ldots$ & & & & & \\
\hline BP & {$[-0.410 ;-0.094]$} & $\ldots$ & & & & \\
\hline FP & {$[0.209 ; 0.520]$} & {$[-0.351 ;-0.010]$} & $\ldots$ & & & \\
\hline HI & {$[0.139 ; 0.483]$} & {$[-0.409 ;-0.121]$} & {$[0.130 ; 0.453]$} & $\ldots$ & & \\
\hline RD & {$[0.090 ; 0.464]$} & {$[-0.332 ;-0.055]$} & {$[0.180 ; 0.471]$} & {$[0.100 ; 0.501]$} & $\ldots$ & \\
\hline UP & {$[0.413 ; 0.717]$} & {$[-0.313 ;-0.002]$} & {$[0.184 ; 0.481]$} & {$[0.208 ; 0.547]$} & {$[0.188 ; 0.601]$} & $\ldots$ \\
\hline
\end{tabular}


Por otro lado, la fiabilidad de la consistencia interna del instrumento se estimó por medio del alfa de Cronbach, en vista que es una herramienta en la que se asume que los ítems (escala tipo Likert) miden a un mismo constructo y están altamente correlacionados (Frías-Navarro, 2013). De esto se interpreta que, si el valor del alfa de Cronbach es más cercano a 1, es mayor la consistencia interna de los ítems, ya que, la prueba alcanza valores positivos comprendidos entre 0 y 1 , donde 0 indica ausencia total de consistencia interna, y 1 , la redundancia total entre los ítems. Para evaluar este coeficiente, Frías-Navarro (2013) sugiere algunos criterios, delimitando que un Alfa de Cronbach $>0.9$ es excelente; $>0.8$ es bueno; $>0.7$ es aceptable; $>0.6$ es cuestionable; $>0.5$ es pobre y $<0.5$ es inaceptable. Así, el instrumento de medida parece tener una adecuada confiabilidad de la consistencia interna, pues toda el alfa de Cronbach se encuentra entre el rango de valores recomendados por los autores mencionados previamente.

Los resultados del análisis confirmatorio evidencian la existencia de un modelo factorial sustentable para el análisis fundamentado en la percepción que los estudiantes de pregrado tienen del trabajo de investigación, el cual es un requisito para graduarse. La presencia de validez convergente y discriminante dentro del instrumento, junto con una confiabilidad aceptable, ratifica que el instrumento evalúa variables fundamentales que inciden directa o indirectamente sobre las experiencias de los estudiantes encuestados en la investigación.

\section{Análisis de resultados y contraste de hipótesis}

Se recogen las diversas hipótesis planteadas y se mide su grado de asociación por medio del estadístico $D$ de Somers. Este coeficiente corresponde a una medida de asociación entre dos variables ordinales que toma un valor comprendido entre -1 y 1 , donde los valores próximos a 1, en valor absoluto, indican una fuerte relación entre las dos variables y los valores próximos a cero indican que hay poca o ninguna relación entre las dos variables (Valencia-Arias y Montoya, 2020). Así mismo, el coeficiente D de Somers fue extraído del software SPSS y ubicado en una tabla de factores cruzados, para que fuera posible observar el grado de asociación entre los factores del modelo; en la tabla 4 evidencia todas las relaciones establecidas entre los constructos del modelo propuesto.

Tabla 4: D de Somers

\begin{tabular}{|c|c|c|c|c|c|c|}
\hline & AV & BP & FP & HI & RD & UP \\
\hline AV & 1.000 & & & & & \\
\hline BP & -0.232 & 1.000 & & & & \\
\hline FP & 0.332 & -0.166 & 1.000 & & & \\
\hline HI & 0.297 & 0.241 & 0.282 & 1.000 & & \\
\hline RD & 0.249 & -0.166 & 0.489 & 0.418 & 1.000 & \\
\hline UP & 0.551 & -0.140 & 0.412 & 0.421 & 0.481 & 1.000 \\
\hline
\end{tabular}

A continuación, en la Figura 1 se presenta el modelo obtenido después de efectuar el análisis factorial exploratorio con sus respectivos valores de asociación entre los factores donde las flechas indican las hipótesis nulas que se validaron en la investigación. En este sentido, con base en lo anteriormente expuesto, uno de los aspectos más reveladores al evaluar la percepción estudiantil frente al desarrollo del trabajo de grado fue entender que el factor que correspondía a las barreras percibidas por el estudiante para la culminación del trabajo de grado, presenta correlaciones negativas con todos los factores tenidos en cuenta en el modelo y con el único que comparte una relación de bajo grado es con el que involucra las habilidades investigativas del estudiante, dejando ver que, las barreras están determinadas por las características individuales de él y su esquema cognitivo, y no tienen nada que ver con el papel del docente como guía y motivador, ni está condicionado por los factores institucionales o curriculares; es decir, solo depende del estudiante y su disposición para aprender.

Los resultados obtenidos de las relaciones hipotéticas muestran que, los aprendizajes que permanecerán a lo largo de la vida del estudiante tienen una influencia positiva frente a la utilidad que este le asigna al trabajo de grado como elemento complementario y necesario en su formación académica; adicional, el docente deberá ejercer un papel dinámico y fuertemente activo a la hora de dirigir las sesiones de asesoría de trabajo de grado con el estudiante, ya que, según la experiencia del estudiante en dichos espacios, será su percepción de utilidad del trabajo de investigación. La facilidad percibida por el estudiante para desarrollar su proyecto de investigación está directamente relacionada con el rol del docente como instructor y formador, dejando ver que el éxito de un trabajo de grado está concentrado en aquellas figuras que pueden generar una buena experiencia en el estudiante, y a su vez se convierte en la herramienta clave para cambiar o mejorar su percepción sobre la institución y sus líderes, ya que desde aquí se pueden suplir diferentes necesidades e incrementar su grado de satisfacción con el aprendizaje obtenido luego de llevar a cabo un trabajo de grado. 


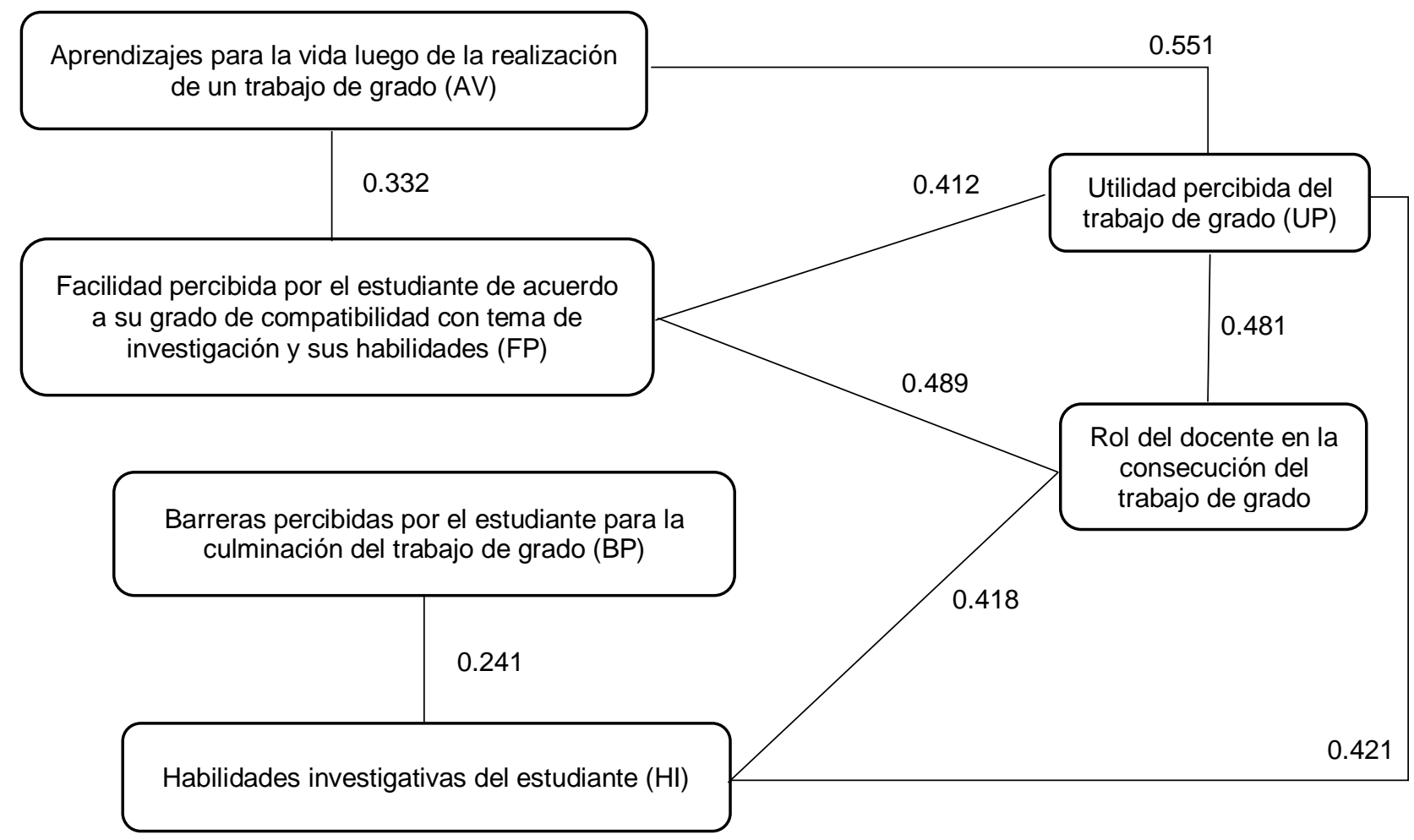

Fig. 1: Modelo propuesto para conocer la percepción de los estudiantes frente a la realización del trabajo de grado

\section{DISCUSIÓN}

Algunas investigaciones han encontrado la incidencia de estos factores internos al explorar la intención de los universitarios por aproximarse a los asuntos científicos, como es el caso de Chara-Saavedra y Olortegui-Luna (2018), quienes en su estudio para determinar los factores asociados a la actitud de estudiantes de enfermería para llevar a cabo ejercicios investigativos, identificaron asuntos como el número de artículos y libros leídos por los participantes, así como el tiempo de dedicación a estas actividades como elementos clave; los cuales, a toda luz hacen parte del fuero interno del individuo.

Frente a aspectos referidos a actores externos -como es el caso de las IES-, el estudio realizado por PulidoMedina et al. (2017), en una asociación de estudiantes de medicina, para la identificación de factores asociados a la intención de llevar a cabo publicaciones científicas demuestra, a diferencia del estudio aquí expuesto, que para la inmersión en actividades de alfabetización científica para los estudiantes sí es relevante el apoyo institucional. De hecho, los autores en su estudio muestran que la reducción del número de publicaciones de los estudiantes está vinculada con la percepción de apoyo de la universidad que éstos poseen. Otras aproximaciones, refieren igualmente aspectos propios de variables exógenas, como es el caso del estudio realizado por De La Cruz-Vargas et al. (2019), quienes muestran la importancia que adquiere la estructuración de cursos en modalidad de taller como estrategia para fortalecer las propuestas de trabajo de grado en estudiantes de medicina del Perú, constituyéndose por tanto en un factor motivacional para la realización de este tipo de trabajos por parte de los estudiantes.

De igual, se ha dejado ver que durante el desarrollo de un tesis participan diferentes personajes con específicas funciones, siendo uno de ellos el director de la tesis, quien se considera que juega un papel de orientador durante el proceso investigativo, es así como se halló un estudio que pretendía analizar algunas percepciones de los estudiantes de maestría sobre los comentarios (observaciones) de sus directores de tesis y como resultado relevante arrojó que los comentarios directos, centrados en el objeto, no en la persona, con sugerencias de solución, son considerados positivos mientras que, los comentarios ambiguos, ofensivos, dilatados en el tiempo y centrados solo en la forma se perciben como poco constructivos, lo anterior se consideró un factor que influye en estimulación del investigador a continuar con su tesis (Ochoa-Sierra y Moreno-Mosquera, 2017).

También, se evidenció un estudio sobre actitudes de los estudiantes hacia la investigación en la Universidad de Ciencias Médicas de Mazandaran. Los resultados mostraron que había una diferencia insignificante entre las variables de edad, género y nivel de educación, mientras que la disponibilidad de suficiente personal de consultoría, asesoramiento, para proporcionar las instalaciones y la motivación de los miembros de la facultad 
y los administradores hacia la investigación puede reducir las barreras hacia investigación del estudiante y tener un impacto positivo en las actitudes hacia la misma (Siamian et al., 2016). Bajo este mismo grupo poblacional, hay otros autores que plantean entre las barreras de realizar investigaciones, no contar con acceso a base de datos científicas, con supervisores profesionales, falta de cursos de capacitación, falta de tiempo, falta de financiación y sensibilización a la investigación (Kumar et al. 2019; Chellaiyan, et.al, 2019), también, se encuentra otra variable en un grupo de estudiantes de farmacia, no tener confianza en sus capacidades para llevar a cabo procesos investigativos (Kritikos et al., 2015).

Por otra parte, en el ejercicio de discusión frente a la actitud de los estudiantes hacia los procesos de investigación científica, Molina-Caballero et al. (2017) llevaron a cabo un estudio con población escolar del grado once en la ciudad de Bogotá, identificando barreras cognitivas para el aprendizaje de la ciencia en esta población de referencia, que obedece a los significados sociales que existen en torno a la dificultad de este tipo de procesos; además de haber identificado falencias en la consolidación de la cultura científica, lo que articulado a los resultados aquí expuestos podría dar lugar a nuevas líneas de investigación en torno a la temática abordada.

Finalmente, es destacable el valor de la enseñanza para capacitar a científicos jóvenes, la información suministrada en el presente artículo podrá ser de utilidad para los investigadores que se desempeñen como asesores y líderes de semilleros de investigación, dado que al identificar los factores que favorecen el desarrollo de proyectos de investigación en estudiantes universitarios, ayudará a brindar un mejor apoyo a sus estudiantes (Feller, 2018; Faber et al., 2020) y definir nuevas estrategias de formación (AlGhamdi et al., 2014), atender inequidades en la investigación, que mejoren la experiencia investigativa en este nivel de formación, más cuando investigaciones han demostrado la relación entre esta variable y la decisión de desarrollar un programa de formación posgradual (Bangera y Brownell, 2014).

\section{CONCLUSIONES}

De acuerdo con el trabajo presentado y a los resultados obtenidos, se pueden plantear las siguientes conclusiones principales:

1) Se logra develar la relevancia de la temática abordada, especialmente de cara al reconocimiento del fomento de la formación investigativa como uno de los pilares del ecosistema de ciencia, tecnología e innovación del país; por lo que incentivar la realización de trabajos de grado por parte de los estudiantes en las Instituciones de Educación Superior -IES-, se constituye en un asunto relevante no sólo para fortalecer esta dimensión de la operación de las IES, sino también como una medida para proveer competencias en los futuros profesionales a egresar al mercado laboral, que les permitan contribuir al desarrollo económico y social del país.

2) Al analizar los factores que favorecen la intención de llevar a cabo proyectos de investigación por parte de estudiantes universitarios, tomando como caso de estudio la ciudad de Medellín; pudo identificarse que éstos están determinados por aspectos propios del estudiante -endógenos-, sin sujeción a variables externas a este como las políticas institucionales, diseños curriculares o el rol de los docentes. Lo cual plantea diferentes reflexiones para las IES en función de la forma como deben orientarse los procesos de formación investigación, al exigir la formulación de políticas que exploren en profundidad las estrategias que deberán ser empleadas para fortalecer capacidades internas del individuo.

3) Finalmente, es necesario explorar los factores constitutivos del fuero interno de las personas y que pueden ser abordados desde las IES, fomentando el desarrollo de una competencia que corresponda a las dinámicas globales actuales y a un elemento diferenciador de los nuevos profesionales que egresan para vincularse al mercado laboral.

\section{REFERENCIAS}

Aiquipa, J., Ramos, C. M., Curay, R., y Guizado, L. L., Factores implicados para realizar o no realizar tesis en estudiantes de psicología, https://doi.org/10.20511/pyr2018.v6n1.180, Propósitos y Representaciones, 6(1), 21-82 (2018).

Alarco, J., Changllio G., y Cahuana M., Investigación en pregrado: interés según sexo y ciclo académico, https://doi.org/10.1016/j.edumed.2016.04.004, Educación médica, 18(1), 67-73 (2017).

AIGhamdi K., Moussa N., y otros tres autores, Perceptions, attitudes and practices toward research among senior medical students, https://doi.org/10.1016/j.jsps.2013.02.006, Saudi Pharmaceutical Journal, 22(2), 113-117 (2014).

Bagozzi, R.P., y Yi, Y., On the evaluation of structural equation models, https://doi.org/10.1007/BF02723327, JAMS 16, 74-94 (1988). 
Bangera, G., y Brownell, S. E., Course-based undergraduate research experiences can make scientific research more inclusive, https://doi.org/10.1187/cbe.14-06-0099, CBE-Life Sciences Education, 13(4), $602-606$ (2014).

Basantes, A., Naranjo, M. E., Gallegos, M. C., y Benítex, N. M., Los dispositivos móviles en el proceso de aprendizaje de la facultad de educación ciencia y tecnología de la universidad técnica del Norte de Ecuador, https://doi.org/10.4067/S0718-50062017000200009, Formación Universitaria, 10(2), 79 - 88 (2017).

Calvo-Porral, C., Martínez-Fernández, V. A., y Juanatey-Boga O., Análisis de dos modelos de ecuaciones estructurales alternativos para medir la intención de compra, ISSN: 0257-4306, Revista Investigación Operacional, 34(3), 230-243 (2013).

Chara-Saavedra, P., y Olortegui-Luna, A., Factores asociados a la actitud hacia la investigación en estudiantes universitarios de enfermería, https://doi.org/10.35626/casus.2.2018.73, CASUS, 3(2), 83-88 (2018).

Chellaiyan, V. G., Manoharan, A., Jasmine, M., y Liaquathali, F., Medical research: Perception and barriers to its practice among medical school students of Chennai, https://doi.org/10.4103/jehp.jehp_464_18, Journal of education and health promotion, 8, 134 (2019).

De La Cruz-Vargas, J. A., Correa-López, L. E., Alatrista-Gutierrez, M. S., y Sánchez Carlessi, H. H., Promoviendo la investigación en estudiantes de medicina y elevando la producción científica en las universidades: experiencia del curso taller de titulación por tesis, ISSN: 1575-1813, Educación Médica, 20(4), 199-205 (2019).

de la Fuente Arias, J., y Justicia Justicia, F., Escala de Estrategias de aprendizaje ACRA-Abreviada para alumnos universitarios, ISSN: 1696-2095, Electronic journal of research in educational psychology 1(2), 139-158 (2003).

Faber, C. J., Kajfez, R. L., y otros cinco autores. Undergraduate engineering students' perceptions of research and researchers, https://doi.org/10.1002/jee.20359, Journal of Engineering Education, 109(4), 780-800 (2020).

Feller M. B., The value of undergraduate teaching for research scientists, https://doi.org/10.1016/j.neuron.2018.09.005, Neuron, 99(6), 1113-1115 (2018).

Frías-Navarro, D., Alfa de Cronbach y consistencia interna de los ítems de un instrumento de medida. Facultad de Psicología. Universidad de Valencia (2013).

García, M. J., Eguia, I., Etxeberria, P., y Alberdi, E., Implementación y evaluación de actividades interdisciplinares mediante applets dinámicas para el estudio de la geometría, http://dx.doi.org/10.4067/S0718-50062020000100063, Formación Universitaria, 13(1), 63-70 (2020).

Gómez-Molina, S., Palacios-Moya, L., y otros cuatro autores. Modelo de satisfacción de egresados universitarios: un estudio de caso, https://doi.org/10.22430/24223182.1443, Revista CEA, 5(10), 49-68 (2019).

Kirillova, K., y Au, W. C. How do tourism and hospitality students find the path to research?, https://doi.org/10.1080/15313220.2020.1713965, Journal of Teaching in Travel \& Tourism, 20(4), 284-307 (2020).

Krim, J. S., Coté, L. E., y otros doce autores, Models and Impacts of Science Research Experiences: A Review of the Literature of CUREs, UREs, and TREs, https://doi.org/10.1187/cbe.19-03-0069, CBE life sciences education, 18(4), $\operatorname{ar65}(2019)$.

Kumar, J., Memon, A., y otros cuatro autores, Barriers experienced by medical students in conducting research at undergraduate level, https://doi.org/10.7759/cureus.4452, Cureus, 11(4), e4452 (2019).

Kritikos, V. S., Saini, B., y otros tres autores, Factors influencing pharmacy students' attitudes towards pharmacy practice research and strategies for promoting research interest in pharmacy practice, https://doi.org//10.18549/PharmPract.2015.03.587, Pharmacy Practice (Granada), 13(3) (2015).

Moeed, A., Science Investigation: Student views about learning, motivation and assessment, $1^{\mathrm{a}}$ edición, https://doi.org/10.1007/978-981-287-384-2, Berlin, Germany: Springer (2015).

Molina-Caballero, M. F., Casas-Mateus, J., y Rivera-Rodríguez, J. C., Actitudes hacia la ciencia en bachilleres de colegios distritales, ISSN: 2500-5324, Revista Latinoamericana De Estudios Educativos, 13(2), 101-121 (2017).

Núñez, J., y Montalvo, L., La Política de Ciencia, Tecnología e Innovación en Cuba y el Papel de las Universidades, Revista Cubana de Educación Superior, ISSN: 0257-4314 34, (1), 29 - 43 (2015).

Ochoa-Sierra, L., y Moreno-Mosquera, E., Some Perceptions of Master's Degree Students on the Comments (remarks) of their Thesis Directors, https://doi.org/10.11144/javeriana.syp36-71.spmd, Signo y Pensamiento, 36(71), 188-206 (2017).

Pulido-Medina, C., Hamon-Rugeles, D. y otros tres autores, Publicación científica entre los directivos de la asociación científica de estudiantes de medicina de Colombia: características y factores asociados,

https://doi.org/10.15446/revfacmed.v65n4.60489, Revista de la Facultad de Medicina, 65(4), 553-557 (2017).

Soria-Barreto, K. L. y Cleveland-Slimming, M. R., Percepción de los estudiantes de primer año de ingeniería comercial sobre las competencias de pensamiento crítico y trabajo en equipo, http://dx.doi.org/10.4067/S0718-

50062020000100103, Formación Universitaria, 13(1), 103-114 (2020). 
Siamian, H., Mahmoudi R. y otros tres autores, Students' attitudes towards research at mazandaran university of medical sciences in 2015, https://doi.org/10.5455/msm.2016.28.468-472, Mater Sociomed. 28(6), 468-472 (2016).

Valencia, J., Macias, J. y Valencia, A. Formative research in higher education: some reflections, https://doi.org/10.1016/j.sbspro.2015.01.562, Procedia - Social and Behavioral Sciences, 176, 940-945 (2015).

Valencia-Arias, A. y Montoya, L., Entrepreneurial intentions among engineering students: applying a theory of planned behavior perspective, https://doi.org/10.3311/PPso.12731, Periodica Polytechnica Social and Management Sciences, 28(1), 59-69 (2020).

Vázquez, Á. y Manassero M., Contenidos de Naturaleza de la Ciencia y la Tecnología en los Nuevos Currículos Básicos de Educación Secundaria, Revista de Currículum y Formación de Profesorado, 21(1), 294-312 (2017). 